Trabajos Originales

\title{
Factores asociados a disfunción sexual en mujeres atendidas en un hospital nivel III-Chiclayo-Perú
}

\author{
Associated factors in women's sexual dysfunction in a hospital level \\ attended III- Chiclayo - Peru
}

\author{
Cristian Díaz-Vélez ${ }^{1,2,3, a}$, Frine Samalvides-Cuba ${ }^{3, b}$
}

1. Hospital Nacional Almanzor Aguinaga Asenjo, EsSalud, Chiclayo, Perú.

2. Centro de Investigación en Epidemiología Clínica y Medicina Basada en Evidencias, Universidad de San Martín de Porres, Chiclayo, Perú

3. Facultad de Medicina Alberto Hurtado. Universidad Peruana Cayetano Heredia, Lima, Perú.

A. Médico Epidemiólogo, Maestro en Epidemiología Clínica.

B. Magister en Epidemiología Clínica.

\section{RESUMEN}

Objetivo: Identificar los factores asociados a la disfunción sexual en las mujeres atendidas en un hospital nivel III. Material y Método: estudio de casos y controles emparejados por edad e índice de masa corporal, realizada en mujeres de 18-59 años que acuden a consultorio externo. Se usó el Índice de Función Sexual Femenina para definir caso ( $\geq 24$ puntos) y control (<24 puntos), y los factores a evaluar fueron síndrome metabólico definida según International Diabetes Federation (IDF), ansiedad y depresión mediante test validados; menopausia, alcohol, fumar, situación de salud, estado civil, grado de instrucción, ocupación, número de embarazos. Resultados: participaron 342 mujeres (114 casos/228 controles), con edad promedio de 40,5 $\pm 9,2$ e IMC de 25,2 $\pm 3,5 ; 45,9 \%$ (157) son casadas; 53,5\% (183) con nivel secundaria, 41,5\% (142) amas de casa, 17,8\% (61) menopaúsicas, 34,8\% (119) con síndrome metabólico. Además, con enfermedad crónica 32,7\% (112), ansiedad 16,4\% (56), depresión 5\% (17), consumo de medicamentos 35,1\% (72). En el análisis bivariado los factores estado civil viuda, ocupación jubilada, 2 embarazos previos, estado de salud enferma, enfermedad crónica, fumar, ansiedad y depresión, y como factor protector, el estado civil casada y nivel de instrucción superior como factores asociados. En el análisis multivariado realizada con regresión logística, se obtuvieron como factores asociados al estado civil casada 0,58 (IC95\%: 0,33-0,87), conviviente 8,11 (IC95\%: 2,36-17,84), fumar 2,26 (IC95\%: 1,08-4,76), ansiedad 1,21 (IC95\%: 1,10-1,45) y estado de salud enfermo 1,50 (IC95\%: 1,261,94). Conclusiones: la disfunción sexual femenina está asociada con estado civil casada/conviviente, ansiedad, estado de salud y fumar.

PALABRAS CLAVE: disfunción sexual, síndrome metabólico, calidad de vida.

\section{ABSTRACT}

Objective: To identify the factors associated with sexual dysfunction in women treated at a level III hospital. Material and Method: case-control study matched by age and body mass index, performed in women aged 1859 years who attend an outpatient clinic. The Female Sexual Function Index was used to define case ( $\geq 24$ points) and control (<24 points), and the factors to be evaluated were International Diabetes Federation (IDF) - 
defined metabolic syndrome, anxiety and depression using validated tests; Menopause, alcohol, smoking, health status, marital status, educational level, occupation, number of pregnancies. Results: 342 women (114 cases / 228 controls) participated, with a mean age of $40.5 \pm 9.2$ and a BMI of $25.2 \pm 3.5 ; 45.9 \%$ (157) are married; $53.5 \%$ (183) with secondary level, $41.5 \%$ (142) housewives, $17.8 \%$ (61) menopausal, 34.8\% (119) with metabolic syndrome. In addition, with chronic disease, $32.7 \%$ (112), anxiety $16.4 \%(56)$, depression $5 \%$ (17), drug consumption $35.1 \%(72)$. In the bivariate analysis, the factors of widowhood, retired occupation, 2 previous pregnancies, ill health, chronic illness, smoking, anxiety and depression, and as a protective factor, married marital status and higher educational level as associated factors. In the multivariate analysis carried out with logistic regression, 0.58 (95\% Cl: 0.33-0.87), associated with marital status, 8.11 (95\% Cl: 2.36-17.84), Smoking 2.26 (95\% Cl: $1.08-4.76)$, anxiety 1.21 (95\% Cl: 1.10-1.45) and ill health status 1.50 (95\% Cl: 1.26- 1.94). Conclusions: Female sexual dysfunction is associated with marital / social status, anxiety, health status and smoking.

KEYWORDS: sexual dysfunction, metabolic syndrome, quality of life

\section{INTRODUCCIÓN}

La sexualidad en la mujer es el reflejo de su nivel de bienestar físico, psíquico y social (1), siendo la disfunción sexual la dificultad o imposibilidad del individuo de participar en las relaciones sexuales tal como lo desea (2). La disfunción sexual femenina (DSF) anteriormente denominado trastorno del deseo sexual hipoactivo en el DSM-IV de la Asociación Americana de Psiquiatría (3) y trastorno del interés y la excitación sexual femenina en la 5ta Edición del DSM (DSM-5) tiene parámetros para la valoración, quedando dividido en 4 dominios: deseo, excitación, orgasmo y dolor(4).

Existen estudios realizados en Estados Unidos, uno de ellos en 31581 mujeres mayores de 18 años (con edad promedio de 49 años), encontró disfunción sexual en $9,5 \%$ (5), mientras que otro realizado en 2 207 mujeres entre 30 y 70 años refiere una prevalencia de $8,3 \%$, y otros estudios muestran tendencias similares con prevalencias entre 7,4\% (6) y $9 \%$ (7); mientras que la prevalencia en mujeres de mediana edad en países Latinoamericanos refieren entre el $25 \%$ y $43 \%$ (8) siendo por ejemplo en Cuba $20,8 \%$ (9) estudio realizado en mujeres de 15 a 59 años de edad que acudieron a consulta externa de un hospital y en Chile $35,6 \%$ (10) estudio realizado en 412 mujeres de 40 a 59 años de edad que acudieron a centros de salud, aunque otras investigaciones refieren que aproximadamente $40 \%$ de las mujeres experimentan algún tipo de problema sexual en el transcurso de su vida (5), lo que la ha convertido en un problema de salud pública.
Los casos de DSF se identifican con más frecuencia en el estado peri y postmenopáusico; llegando a ser entre el $50 \%$ (11) en un estudio realizado en mujeres de 40 a 64 años de edad que acudieron a consulta 0 como acompañantes y $65,6 \%$ (8) de mujeres de 45 a 59 años de edad en estado menopaúsico, aumentando aún más con la edad $(12,13)$; debido a que el $40 \%$ reportan la pérdida de placer sexual y dificultad para alcanzar el orgasmo (14), además de otros problemas frecuentes como lubricación y dolor durante el coito (15).

Los factores asociados a la disfunción sexual han sido estudiados por varios autores, registrando el síndrome metabólico como un factor de riesgo independiente (16-18), la edad, el grado de instrucción, la salud física $(19,20)$ y mental en la mujer (21), aunque otros autores refieren además el estado civil casado, hábito de fumar (22), así como tener más de 14 años de matrimonio, más de 2 hijos, el grado de instrucción, el tener una pareja mayor de 42 años (23) y el uso de terapia antidepresiva (24).

A nivel latinoamericano existen pocos estudios realizados, uno de ellos en mujeres mexicanas, reportándose como factores asociados la edad mayor a 40 años, el laborar fuera del hogar, la presencia de obesidad y/o sobrepeso, presentar hipertensión arterial, diabetes mellitus, haber presentado histerectomía previa, la maternidad, la disfuncionalidad sexual conyugal, la depresión, familia disfuncional, estrato socioeconómico bajo (25); otra investigación en mujeres colombianas nos refiere que el hábito de fumar, la hipertensión arterial como factores de riesgo, mientras que escolaridad mayor de diez años sería un factor protector (26); mientras que 
una investigación realizada en mujeres chilenas refieren el estado menopáusico, la edad y la terapia de reemplazo hormonal como factores asociados (11), aspectos que pueden llegar a afectar considerablemente la calidad de vida de la mujer, en el contexto familiar, social y laboral (27).

A nivel nacional no se hemos encontrado estudios de los factores asociados a disfunción sexual femenina, y dada la importancia de conocer estos factores planteamos realizar el estudio para identificarlos, por lo cual nos planteamos el objetivo de identificar los factores asociados a la disfunción sexual en las mujeres atendidas en un hospital nivel III. ChiclayoPerú.

\section{MATERIAL Y METODOS}

\section{DISEÑO DEL ESTUDIO:}

El diseño es estudio de casos y controles con emparejamiento individual por edad e índice de masa corporal.

\section{POBLACIÓN:}

Se incluyó a mujeres que acuden a consultorio externo del Hospital Nacional Almanzor Aguinaga Asenjo.

- CASOS

Toda mujer con disfunción sexual definida como un puntaje menor o igual a 24 en el Índice de Función Sexual Femenina (IFSF) (1).

\section{- CONTROLES}

Toda mujer sin disfunción sexual definida con puntaje mayor de 24 en el Índice de Función Sexual Femenina (IFSF) (1).

\section{CRITERIOS DE SELECCIÓN}

\section{CRITERIOS DE INCLUSIÓN}

- CASOS

- Mujeres que realizan sus actividades rutinarias definido por la encuesta de Vida Social y Salud (28).

- $\quad$ Mujeres de 18 a 59 años atendidas en consultorio externo de oftalmología, otorrinolaringología y dermatología.

- CONTROLES

- Mujer sin disfunción sexual emparejado por edad e IMC.
Mujeres que realizan sus actividades rutinarias definido por la Encuesta de Vida Social y Salud (28).

Mujeres de 18 a 59 años atendidas en consultorio externo de oftalmología, otorrinolaringología y dermatología.

\section{CRITERIOS DE EXCLUSIÓN PARA CASOS Y CONTROLES}

Mujeres con déficit mental que les impida entender el cuestionario.

Mujeres gestantes o 2 meses en periodo postparto.

- Mujeres con ceguera u otra discapacidad reconocida de los órganos de los sentidos

- Mujeres con accidente cerebro vascular secuelar o lesión de médula espinal

- Mujeres con neoplasia maligna, amputación alta de miembros inferiores

Mujeres con insuficiencia renal crónica terminal en plan de hemodiálisis.

- $\quad$ Mujeres que no acepten participar del estudio.

\section{DEFINICIÓN DE VARIABLES}

\section{VARIABLE DEPENDIENTE}

- Disfunción sexual: según en el Índice de Función Sexual Femenina (IFSF), es puntaje de menor o igual a 24. Utilizándose un instrumento de 19 ítems, con puntuación, basada en 6 principales componentes: deseo, excitación, lubricación, orgasmo, satisfacción y dolor, validado al castellano, el sistema de puntuación del IFSF es el resultado de puntuaciones de preguntas individuales y de los dominios, multiplicados por el factor correspondiente(1).

\section{VARIABLES INDEPENDIENTES}

- Síndrome metabólico: según International Diabetes Federation Consensus Panel (IDF)(29)

- Obesidad abdominal: criterio indispensable que se fija a $\geq 94 \mathrm{~cm}$ en varones $\mathrm{y} \geq 80 \mathrm{~cm}$ mujeres.

Presión arterial: valores $\geq 130 / 85 \mathrm{mmHg}$ para ambos sexos, o que esté recibiendo tratamiento antihipertensivo.

Triglicéridos en ayunas: valores sanguíneos $\geq 1,70 \mathrm{mmol} / \mathrm{L} \quad(\geq 150 \mathrm{mg} / \mathrm{dl})$, o que esté 
recibiendo tratamiento farmacológico para la hipertrigliceridemia.

- c-HDL en ayuna: valores sanguíneos $<1,04$ $\mathrm{mmol} / \mathrm{L} \quad(<40 \mathrm{mg} / \mathrm{dl})$ en los hombres y $<1,29$ $\mathrm{mmol} / \mathrm{L}(<50 \mathrm{mg} / \mathrm{dl})$ en las mujeres, o que esté recibiendo tratamiento farmacológico para la hipercolesterolemia.

- $\quad$ Glicemia en ayunas: valores plasmáticos $\geq 5,6$ $\mathrm{mmol} / \mathrm{L}$ ( $\geq 100 \mathrm{mg} / \mathrm{dl}$ ) para ambos sexos; o diabetes mellitus preexistente.

- Depresión: medida con la escala hospitalaria validada de HAD (Hospital Anxiety and Depression Scale) se considera depresión si la puntuación de cada ítem es de 0 a 3, entendiendo que una puntuación por encima de 11 es indicativa de sintomatología depresiva(30)

- Ansiedad: medida con la escala hospitalaria validada de HAD (Hospital Anxiety and Depression Scale) se considera ansiedad la puntuación de cada ítem es de 0 a 3, entendiendo que una puntuación por encima de 11 es indicativa de sintomatología ansiosa(30).

- $\quad$ Estado menopausico: (amenorrea > 12 meses) aquellas mujeres histerectomizadas con ooforectomía bilateral serán consideradas como post-menopáusicas. Las mujeres histerectomizadas con ovarios conservados (uno o los dos) no serán consideradas en este grupo(31).

- Consumo de alcohol y hábito de fumar: se evaluó con preguntas tomadas de la encuesta demográfica y de salud familiar 2012(32).

- Percepción del estado de salud: presencia de enfermedad crónica, antecedente de intervenciones quirúrgicas y consumo de medicamentos.

- Factores epidemiológicos y socioeconómicos: en el estudio se utilizaron el estado civil (soltera, casada, viuda y conviviente), el grado de instrucción (primaria, secundaria y superior), la ocupación (empleada, desempleada, jubilada, ama de casa) y el número de embarazos.

\section{CALCULO DEL TAMAÑO MUESTRAL MUESTRA}

El estudio se realizó en mujeres aseguradas en Seguro Social EsSalud, realizándose el cálculo muestral con el programa Epidat versión 3.1 para un estudio de casos y controles emparejado con un $\mathrm{p} 1$ (proporción de pacientes con síndrome metabólico con disfunción sexual igual a 36\%) (33) y p2 (proporción de pacientes sin síndrome metabólico con disfunción sexual igual a 21\%) (33), con OR>2, con una potencia de $80 \%$, un nivel de confianza de $95 \%$ y 2 controles para cada caso; obteniéndose 114 para los casos y 228 para los controles.

\section{MUESTREO}

Se realizó muestreo probabilístico por conglomerados monoetápico aleatorio, se seleccionaron al azar del listado de personas citadas a consultorio externo.

- Conglomerados: días fijados para consultorio externo de los 3 servicios seleccionados. De ellos se escogió al azar 5 días del mes durante 16 meses.

- Aleatorio: de cada día seleccionado se obtenía la lista del Sistema de Gestión Hospitalaria, y en forma aleatoria se obtenía 3 pacientes mujeres.

\section{PLAN DE ANALISIS}

Se realizó análisis estadístico descriptivo con medidas de centralización y dispersión para las variables cuantitativas. Asimismo, se calculó frecuencias absolutas y relativas para las variables cualitativas. También se aplicó estadística inferencial entre los grupos de estudio y cada uno de los factores a estudiar con Odds Ratio (OR), usando intervalos de confianza al $95 \%$. Finalmente se realizó análisis multivariado con regresión logística con un nivel de confianza de $95 \%$ y nivel de significancia de $p<0,05$. Se evaluó el modelo multivariado con la prueba de bondad de ajuste de Hosmer-Lemeshow y AIC.

\section{CONSIDERACIONES ETICAS}

El trabajo de investigación fue aprobado por el Comité de Ética y Comité de Investigación del Hospital Nacional Almanzor Aguinaga Asenjo (HNAAA) y del Comité de Ética de la Universidad Peruana Cayetano Heredia e inscrito con código SIDISI: 57164.

\section{RESULTADOS}

Participaron en el estudio 342 mujeres siendo 114 casos y 228 controles, con una edad de 40,5 $\pm 3,2$ e índice de masa corporal de 25,2 $\pm 3,5$. El estado civil casada fue el más frecuente con 45,9\% (157); el grado de instrucción nivel secundario con 53,5\% (183), mientras que la ocupación más referida fue ama de casa con 41,5\% (142). Las frecuencias de los factores asociados nos muestran a mujeres en estado menopaúsico en 17,8\% (61), con síndrome metabólico $34,8 \%$ (119), la presencia de enfermedad 
crónica a $32,7 \%$ (112), el riesgo de ansiedad 16,4\% (56), el riesgo de depresión 5\% (17).

En el análisis bivariado descriptivo según la presencia de la DSF se encontró que el 62,3\% (71) de los casos y $50 \%$ (114) de los controles no eran casadas; el grado de instrucción más frecuente fue nivel secundario para ambos grupos, 56,1\% (64) en casos y $52,2 \%$ (119) en controles respectivamente. En la ocupación, ser ama de casa fue más frecuente en los casos con $43,9 \%$ (50) y empleada $47,4 \%$ (108) en los controles; y el estado de menopausia se presentó en $22,8 \%$ (26) en los casos y $14,5 \%$ (33) en los controles. Respecto al haber tenido por lo menos una gestación el $79,8 \%$ (91) de los casos y 73,3\% (167) de los controles lo refirieron, siendo más frecuente 2 o más hijos con $66,7 \%$ (76) en los casos y $51,8 \%$ (118) en los controles. En otros factores estudiados encontramos que el síndrome metabólico se presentó en el $37,7 \%$ (43) de los casos y $33,3 \%$ (76) en los casos y controles respectivamente, habiendo referido los primeros un estado de salud enfermo en $56,1 \%$ (64) y los controles $28,1 \%$ (64). Los casos refirieron riesgo de ansiedad en $34,2 \%$ (39), el riesgo de depresión en $8,8 \%$ (10); en los controles refirieron riesgo de ansiedad en $7,5 \%$ (17), el riesgo de depresión en 3,1\% (7) (Ver Tabla 01).

En el análisis bivariado se obtuvieron entre los principales factores asociados el estado civil viudez con un OR de 5,91 (IC95\%:2,44-14,27, p<0,05) y la ansiedad con un OR de 6,45 (IC95\%3,46-12,01, $\mathrm{p}<0,05)$, el hábito de fumar con OR de 3,45 (IC95\%:1,23-4,67, $\mathrm{p}<0,05)$ y como factores protectores el estar casada con un OR de 0,60 (IC95\%:0,38-0,95, p<0,05) y grado de instrucción superior con OR de 0,50 (IC95\%: 0,32-0,80, p<0,05) (Ver Tabla 02).

En el análisis multivariado se mantuvieron como factores de riesgo el estado civil de viudez en 8,11 (IC95\%: 2,36-17,84, p<0,05), la ansiedad en 1,21 (IC95\%: 1,10-1,45, p<0,05), la percepción del estado de salud como enferma en 1,50 (IC95\%: 1,26-1,94, $\mathrm{p}<0,05)$ y el hábito de fumar en 2,26 (IC95\%: 1,08$4,76, p<0,05)$ y como factor protector el estado civil casada 0,58 (IC95\%: 0,33-0,87, p<0,05), que nos dice que disminuye en $52 \%$ la probabilidad de tener el antecedente de disfunción sexual (Ver Tabla 03).

\section{DISCUSIÓN}

La mayor proporción, tanto en casos y controles, son mujeres casadas concordante a los hallazgos de Pérez-Martínez C. et. al.(33) que menciona que sólo $43,2 \%$ de las mujeres casadas refieren una función sexual adecuada y Blümel J. et. al. refirieron que el $68 \%$ de las mujeres que dejaban de tener actividad sexual, eran casadas(34). Asimismo, mencionar que el estado conyugal de casada es el más frecuente en la población peruana con una 30,8\% (INEI, 2014). Otra característica sociodemográfica frecuente encontrada fue el grado de instrucción secundaria, semejante a lo que ocurre en el Perú; donde el 32,3\% de las mujeres mayores de 25 años tiene educación secundaria, seguida de $28,9 \%$ para educación primaria (INEI, 2014). Además, tener 2 hijos o más fue la condición de paridad más frecuente tanto en los casos y controles y según Figueroa R. et. al. (10) refiere un promedio de $2,8 \pm 1,5$ hijos en mujeres con disfunción sexual.

Entre los factores estudiados no se encontró asociado al síndrome metabólico, no concordando con el estudio realizado en Piura-Perú (35) que encontraron asociación con diabetes mellitus en los primeros años postmenopausia, aunque cabe mencionar que la diabetes mellitus podría ser parte de la definición de síndrome metabólico; lo que estaría solapado por los otros componentes del síndrome, además otro estudio lo asocian a deseo sexual (17)que en nuestro estudio fue medido sólo en una dimensión del instrumento. Debemos tener en cuenta que los criterios diagnósticos del síndrome metabólico han sido sujeto de muchas definiciones, el uso de diferentes definiciones para el diagnóstico, podría condicionar una variación en la frecuencia del síndrome metabólico en la población, según una u otra definición; y claro con ello modificar las asociaciones que esta pueda presentar. Uno de los puntos discordantes en las definiciones es la obesidad abdominal, la IDF considera que debiera ser el principal criterio diagnóstico y excluyente para diagnosticar síndrome metabólico(36). La OMS no lo considera como el criterio principal, y para el ATP III es un componente de los cinco propuestos, pero no excluyente para diagnosticar síndrome metabólico (37); aspectos que pueden haber influenciado en la no presencia de asociación de nuestros hallazgos.

Un estudio realizado en población Chilena (38) menciona que la disfunción sexual en mujeres está asociada con diversos factores psicodemográficos como jubilada, grado de instrucción superior, pobre 
salud física (percepción de enfermedad) y mental (ansiedad y depresión); semejante a los factores encontrados en nuestro estudio donde ansiedad, el estado de salud enferma, fumar; el estado civil de casada y conviviente están asociados. Además existen otros estudios que mencionan a la menopausia asociada a la disfunción sexual(39), pero nuestros resultados no lo evidenciaron; quizás debido a la frecuencia de disfunción sexual en no menopaúsicas que fue $30,9 \%$ comparada a $44,26 \%$ en menopaúsicas (24).

Otras variables estudiadas como comorbilidades ginecológicas, consumo de alcohol y uso de fármacos no se encontraron asociadas a disfunción sexual, sin embargo, se encontró mayor frecuencia de mujeres con disfunción sexual; en quienes consumen hormonas y según Blümel JE. y col. (38) refieren el uso de terapia de reemplazo hormonal está asociado a disfunción sexual.

Kusnetzoff J. (42) describe como las causas más frecuentes de anorgasmia: 1) un conflicto conyugal; 2) una falla orgásmica accidental debida a cansancio, preocupaciones, desconcentración; 3) la ingestión de medicamentos inhibitorios; 4) un estado advertido o no, que probablemente en nuestro estudio se estén presentando pero al no haber sido evaluadas podrían estas ser de mayor importancia; si tomamos en cuenta que según un estudio realizado en 3 regiones del Perú (Lima, Pucallpa y Huancayo) las mujeres refieren haber sufrido de sufre agresión física (45,1\%), maltrato emocional $(58,6 \%)$ y ha tenido o tiene relaciones sexuales no deseadas o por obligación $(28,3 \%)(44)$.

El contar con una tasa de no participación de aproximadamente $25 \%$ de las mujeres inicialmente seleccionadas, podría sesgar el estudio a que probablemente las más enfermas aceptaron ingresar al estudio.

Según los resultados obtenidos se concluye que la disfunción sexual femenina está asociada con el estado civil viuda, riesgo de ansiedad, percepción del estado de salud como enferma y el hábito de fumar; y como factor protector el estado civil casada.

\section{BIBLIOGRAFÍA}

1. Blümel JE, Binfa L, Cataldo $P$, Carrasco $A$, Izaguirre $H$, Sarrá $S$. Índice de función sexual femenina: un test para evaluar la sexualidad de la mujer. Rev Chil Obstet Ginecol. 2004;69(2):11825.

2. Rochera MB. Fisioterapia en las disfunciones sexuales femeninas. Editorial Club Universitario; 2010. 45 p.

3. DSM-IV-TR [Internet]. American Psychiatric Association; 2000 [citado el 23 de septiembre de 2016]. 982 p. (DSM Library). Recuperado a partir de:

http://dsm.psychiatryonline.org/doi/abs/10.1176/a ppi.books.9780890420249.dsm-iv-tr

4. American Psychiatric Association. Diagnostic and Statistical Manual of Mental Disorders [Internet]. Fifth Edition. American Psychiatric Association; 2013 [citado el 23 de septiembre de 2016]. Recuperado a partir de: http://psychiatryonline.org/doi/book/10.1176/appi. books.9780890425596

5. Shifren JL, Monz BU, Russo PA, Segreti A, Johannes CB. Sexual problems and distress in United States women: prevalence and correlates. Obstet Gynecol. 2008;112(5):970-8.

6. Rosen RC, Connor MK, Miyasato G, Link C, Shifren JL, Fisher WA, et al. Sexual desire problems in women seeking healthcare: a novel study design for ascertaining prevalence of hypoactive sexual desire disorder in clinic-based samples of US women. J Womens Health. 2012;21(5):505-15.

7. Leiblum SR, Koochaki PE, Rodenberg CA, Barton IP, Rosen RC. Hypoactive sexual desire disorder in postmenopausal women: US results from the Women's International Study of Health and Sexuality (WISHeS). Menopause. 2006;13(1):4656.

8. Izquierdo MS, Hernández-García E. Climaterio y sexualidad. Enferm Glob. 2015;14(40):76-85.

9. Díaz NE, Ulloa M de los ÁG, Daniel P, Gil P, Cruz WV. Disfunción sexual en un grupo de mujeres del municipio de Güines. Rev Cienc MÉDICAS HABANA [Internet]. 2014 [citado el 23 de septiembre de 2016];20(1). Recuperado a partir de:

http://www.medigraphic.com/pdfs/revciemedhab/c mh-2014/cmh141h.pdf 
10. Figueroa R, Jara D, Fuenzalida A, del Prado M, Flores D, Blumel JE. Prevalencia de disfunción sexual en mujeres climatéricas. Rev Médica Chile. 2009;137(3):345-50.

11. Blümel JE, Araya $H$, Riquelme R, Castro G, Sánchez F, Gramegna G. Prevalencia de los trastornos de la sexualidad en mujeres climatéricas: Influencia de la menopausia y de la terapia de reemplazo hormonal. Rev Médica Chile. 2002;130(10):1131-8.

12. Fierro D, Rechkemmer A, Roman V, Fierro L, Ruiz M. Sexualidad y menopausia. Rev Peru Ginecol Obstet. 2015;43(1):41-8.

13. Castañeda C, Castillo J, Lazo F, Gutiérrez R, Medina M, Casas M. Sexualidad y menopausia en mujeres mayores de 39 años. Rev Peru Ginecol Obstet. 2015;46(3):270-4.

14. Influencia de la menopausia en la funcion sexual [Internet]. [citado el 16 de septiembre de 2016]. Recuperado a partir de: https://encolombia.com/medicina/revistasmedicas/menopausia/vm-101/meno10104menopausia/

15. Rosen RC, Taylor JF, Leiblum SR, Bachmann GA. Prevalence of sexual dysfunction in women: results of a survey study of 329 women in an outpatient gynecological clinic. J Sex Marital Ther. 1993;19(3):171-88.

16. Ponholzer A, Temml C, Rauchenwald M, Marszalek M, Madersbacher $S$. Is the metabolic syndrome a risk factor for female sexual dysfunction in sexually active women? Int J Impot Res. 2008;20(1):100-4.

17. Esposito K, Ciotola M, Marfella R, Di Tommaso D, Cobellis L, Giugliano D. The metabolic syndrome: a cause of sexual dysfunction in women. Int $\mathrm{J}$ Impot Res. 2005;17(3):224-6.

18. Martelli V, Valisella S, Moscatiello S, Matteucci C, Lantadilla C, Costantino A, et al. Prevalence of sexual dysfunction among postmenopausal women with and without metabolic syndrome. J Sex Med. 2012;9(2):43441.

19. Laumann EO, Paik A, Rosen RC. Sexual dysfunction in the United States: prevalence and predictors. Jama. 1999;281(6):537-44.
20. Arcila JFU, Tobón MTQ, Gómez MG. Orgasmo femenino: definición y fingimiento. Urol Colomb. 2015;24(1):19-27.

21. Bakhtiari A, Basirat Z, Nasiri-Amiri F. Sexual Dysfunction in Women Undergoing Fertility Treatment in Iran: Prevalence and Associated Risk Factors. J Reprod Infertil. marzo de 2016;17(1):26-33.

22. Avis NE, Stellato R, Crawford S, Johannes C, Longcope $\mathrm{C}$. Is there an association between menopause status and sexual functioning?. Menopause. 2000;7(5):297-309.

23. Sidi H, Puteh SEW, Abdullah N, Midin M. The prevalence of sexual dysfunction and potential risk factors that may impair sexual function in Malaysian women. J Sex Med. marzo de 2007;4(2):311-21.

24. Yanez D, Castelo-Branco C, Hidalgo LA, Chedraui PA. Sexual dysfunction and related risk factors in a cohort of middle-aged Ecuadorian women. J Obstet Gynaecol. 2006;26(7):682-6.

25. Vega M G, C F, Jezabel P, Ávila M J, Becerril $S \mathrm{~A}, \mathrm{M} \mathrm{V}$, et al. Factores biopsicosociales asociados a la disfunción sexual femenina en una población mexicana. Rev Chil Obstet Ginecol. 2014;79(2):81-5.

26. Castro A de JM, Márquez-Vega J, ArtetaAcosta C. Disfunción sexual en mujeres climatéricas afrodescendientes del Caribe Colombiano. latreia. 2013;27(1):31-41.

27. Li S, Holm K, Gulanick M, Lanuza D. Perimenopause and the quality of life. Clin Nurs Res. 2000;9(1):6-23.

28. Estadística IN de, (Peru) I, (Programme) MIM. Encuesta demográfica y de salud familiar, ENDES Continua 2004: informe principal. Instituto Nacional de Estadística e Informática; 2005.

29. Bello Rodríguez B, Sánchez Cruz G, Ferreira Pinto AC, Báez Pérez EG, Fernández Morín J, Achiong Estupiñan F. Síndrome Metabólico: un problema de salud con múltiples definiciones. Rev Médica Electrónica. 2012;34(2):199-213.

30. López-Roig S, Terol MC, Pastor MA, Neipp MC, Massutí B, Rodríguez-Marín J, et al. Ansiedad y depresión. Validación de la escala HAD en pacientes oncológicos. Rev Psicol Salud. 2000;2(12):127-57. 
31. Sociedad Española de Ginecología. obstetricia, Asociación Española para el estudio de la Menopausia, Sociedad Española de Medicina de familia y Comunitaria y centro Cochrane iberoamericano. Guía Práctica Clínica Menopaus Post Menopaus Barc Mayo. 2004;

32. Instituto Nacional de Estadística e Informática (Peru). Perú encuesta demográfica y de salud familiar: 2012: nacional y departamental. 2013.

33. Pérez-Martínez C, Vargas-Díaz IB, CisnerosCastolo M. Prevalencia de disfunción sexual femenina en una población que asiste a una unidad de urología. 2008 [citado el 16 de septiembre de 2016]; Recuperado a partir de: http://new.medigraphic.com/cgibin/resumen.cgi?IDARTICULO=29338

34. Blümel JE. Disfunción sexual en la mujer chilena. Medwave [Internet]. 2009 [citado el 16 de marzo de 2016];9(10). Recuperado a partir de: http://www.medwave.cl/link.cgi/medwave/congres os/4211

35. Mezones-Holguin E, Blümel JE, Huezo M, Vargas R, Castro J, Córdova W, et al. Impact of diabetes mellitus on the sexuality of Peruvian postmenopausal. Gynecol Endocrinol. 2008;24(8):470-4.

36. Zimmet P, Alberti KGMM, Serrano Ríos M. Una nueva definición mundial del síndrome metabólico propuesta por la Federación Internacional de Diabetes: fundamento y resultados. Rev Esp Cardiol. 2005;58(12):1371-6.

37. Lizarzaburu Robles JC. Síndrome metabólico: concepto y aplicación práctica. En: Anales de la Facultad de Medicina [Internet]. UNMSM. Facultad de Medicina; 2013 [citado el 6 de septiembrede 2015]. p. 315-20. Recuperado a partir de: http://www.scielo.org.pe/scielo.php?pid=S1025$55832013000400009 \&$ script=sci_arttext
38. Blümel JE, Chedraui $P$, Calle A, Bocanera R, Depiano E, Figueroa-Casas $P$, et al. Age at menopause in Latin America. Menopause. 2006;13(4):706-12.

39. Dombek K, Capistrano EJM, Costa ACC, Marinheiro LPF. Risk factors associated with sexual dysfunction in Brazilian postmenopausal women. Int J Impot Res. marzo de 2016;28(2):627.

40. Blümel JE, Castelo-Branco C, Riquelme R, Araya $H$, Jaramillo $P$, Tacla $X$, et al. Use of hormone replacement therapy among Chilean women: a comparison between socioeconomic levels. Menopause. 2002;9(5):377-80.

41. Blümel JE, Chedraui $P$, Baron $G$, Belzares E, Bencosme A, Calle A, et al. Sexual dysfunction in middle-aged women: a multicenter Latin American study using the Female Sexual Function Index. Menopause. 2009;16(6):1139-48.

42. La mujer sexualmente feliz | Contralnfo.Com [Internet]. [citado el 16 de septiembre de 2016]. Recuperado a partir de: http://www.contrainfo.com/5644/la-mujersexualmente-feliz/

43. Kusnetzoff JC. La mujer sexualmente feliz. Ediciones Granica Mexico; 2004.

44. Díaz-Vélez C, Apolaya Segura M, VeraRomero O, Castillo-Mori Y. Impacto y factores de riesgo sobre depresión en mujeres en tres regiones del Perú [Internet]. Lima, Perú: Movimiento Manuela Ramos; 2013. Recuperado a partir de: http://www.manuela.org.pe/impacto-yfactores-de-riesgo-sobre-depresion-en-mujeresen-tres-regiones-del-peru/ 
Tabla 01: Características según presencia de disfunción sexual de las mujeres atendidas en un hospital nivel III.

\begin{tabular}{|c|c|c|c|c|}
\hline \multirow{3}{*}{$\begin{array}{ll} & \text { Característica } \\
\text { Estado Civil }\end{array}$} & \multicolumn{4}{|c|}{ Disfunción Sexual } \\
\hline & \multicolumn{2}{|c|}{ SI } & \multicolumn{2}{|c|}{ NO } \\
\hline & $\mathbf{n}$ & $\%$ & $\mathbf{n}$ & $\%$ \\
\hline Soltera & 35 & $30,7 \%$ & 59 & $25,9 \%$ \\
\hline Casada & 43 & $37,7 \%$ & 114 & $50,0 \%$ \\
\hline Viuda & 18 & $15,8 \%$ & 7 & $3,1 \%$ \\
\hline Conviviente & 18 & $15,8 \%$ & 48 & $21,1 \%$ \\
\hline \multicolumn{5}{|l|}{ Grado de instrucción } \\
\hline Primaria & 7 & $6,1 \%$ & 5 & $2,2 \%$ \\
\hline Secundaria & 64 & $56,1 \%$ & 119 & $52,2 \%$ \\
\hline Superior & 43 & $37,7 \%$ & 104 & $45,6 \%$ \\
\hline \multicolumn{5}{|l|}{ Ocupación } \\
\hline Empleada & 44 & $38,6 \%$ & 108 & $47,4 \%$ \\
\hline Desempleada & 7 & $6,1 \%$ & 7 & $3,1 \%$ \\
\hline Jubilada & 13 & $11,4 \%$ & 11 & $4,8 \%$ \\
\hline Ama de casa & 50 & $43,9 \%$ & 92 & $40,4 \%$ \\
\hline Menopausia & 27 & $23,7 \%$ & 34 & $14,9 \%$ \\
\hline \multicolumn{5}{|l|}{ Número de Embarazos } \\
\hline 0 & 23 & $20,2 \%$ & 61 & $26,8 \%$ \\
\hline 1 & 15 & $13,2 \%$ & 49 & $21,5 \%$ \\
\hline 2 & 35 & $30,7 \%$ & 48 & $21,1 \%$ \\
\hline 3 & 23 & $20,2 \%$ & 44 & $19,3 \%$ \\
\hline 4 & 18 & $15,8 \%$ & 26 & $11,4 \%$ \\
\hline Síndrome Metabólico & 43 & $37,7 \%$ & 76 & $33,3 \%$ \\
\hline \multicolumn{5}{|l|}{ Percepción del estado de salud } \\
\hline Sana & 50 & $43,9 \%$ & 164 & $71,9 \%$ \\
\hline Enferma & 64 & $56,1 \%$ & 64 & $28,1 \%$ \\
\hline Riesgo de ansiedad & 39 & $34,2 \%$ & 17 & $7,5 \%$ \\
\hline Riesgo de depresión & 10 & $8,8 \%$ & 7 & $3,1 \%$ \\
\hline
\end{tabular}


Tabla N02: Análisis bivariado de factores asociados a disfunción sexual de las mujeres atendidas en un hospital nivel III.

\begin{tabular}{|c|c|c|}
\hline Factores & $\begin{array}{c}\text { OR } \\
\text { crudo }\end{array}$ & IC95\% \\
\hline Síndrome Metabólico & 1,21 & $0,75-1,93$ \\
\hline \multicolumn{3}{|l|}{ Estado Civil } \\
\hline Soltera & 1,26 & $0,77-2,08$ \\
\hline Casada & 0,60 & $0,38-0,95$ \\
\hline Viuda & 5,91 & $2,44-14,27$ \\
\hline Conviviente & 0,70 & $0,38-1,26$ \\
\hline \multicolumn{3}{|l|}{ Grado de instrucción } \\
\hline Primaria & 2,91 & $0,95-8,91$ \\
\hline Secundaria & 1,17 & $0,74-1,84$ \\
\hline Superior & 0,50 & $0,32-0,80$ \\
\hline \multicolumn{3}{|l|}{ Ocupación } \\
\hline Empleada & 0,64 & $0,40-1,01$ \\
\hline Desempleada & 1,97 & $0,70-5,53$ \\
\hline Jubilada & 2,42 & $1,06-5,49$ \\
\hline Ama de casa & 1,06 & $0,67-1,68$ \\
\hline Menopausia & 1,74 & $0,98-3,08$ \\
\hline \multicolumn{3}{|l|}{ Número de Embarazos } \\
\hline 0 & 0,69 & $0,40-1,18$ \\
\hline 1 & 0,55 & $0,29-1,03$ \\
\hline 2 & 1,66 & $1,00-2,75$ \\
\hline 3 & 1,05 & $0,60-1,84$ \\
\hline 4 & 1,45 & $0,76-2,76$ \\
\hline Percepción del estado de salud Enferma & 3,28 & $2,05-5,23$ \\
\hline Hábito de fumar & 3,45 & $1,23-4,67$ \\
\hline Riesgo de ansiedad & 6,45 & $3,46-12,01$ \\
\hline Riesgo de depresión & 3,03 & $1,16-7,93$ \\
\hline
\end{tabular}

Tabla N03: Análisis multivariado de factores asociados a disfunción sexual de las mujeres atendidas en un hospital nivel III.

\begin{tabular}{|l|c|c|c|}
\hline \multicolumn{1}{|c|}{ Factores } & OR & \multicolumn{2}{c|}{ IC95\% } \\
\hline Estado Civil (casada) & ajustado & Inferior & Superior \\
\hline Estado Civil (viuda) & 0,58 & 0,33 & 0,87 \\
\hline Riesgo de ansiedad & 8,11 & 2,36 & 27,84 \\
\hline Percepción del estado Salud (enferma) & 1,21 & 1,10 & 1,45 \\
\hline Hábito de fumar & 1,50 & 1,26 & 1,94 \\
\hline
\end{tabular}

Prueba de bondad de ajuste de Hosmer-Lemeshow $>0,05$

$\mathrm{AIC}=123,4$ 